\title{
Bickerstaff's encephalitis
}

\author{
Emma Horton, ${ }^{1}$ Sanjay Krishnamoorthy, ${ }^{2}$ Lucy Reynolds ${ }^{3}$
}

${ }^{1}$ Chelsea and Westminster Hospital, London, UK

${ }^{2}$ Department of Acute Medicine and General Internal

Medicine, Chelsea and Westminster Hospital,

London, UK

${ }^{3}$ Department of Neurology, St George's Hospital, London, UK

\section{Correspondence to}

Dr Emma Horton,

emma.horton07@imperial.ac.uk

Accepted 10 July 2014

\section{SUMMARY}

Bickerstaff's brainstem encephalitis is a rare syndrome defined by the triad of ophthalmoplegia, ataxia and decreased consciousness. It is considered to be a variant of Miller Fisher syndrome and Guillain-Barré syndrome but is differentiated from the two by the presence of central nervous system involvement, commonly in the form of impaired consciousness. We present an unusual case of Bickerstaff's encephalitis, where the patient presented with pseudobulbar affect.

\section{BACKGROUND}

Bickerstaff's brainstem encephalitis (BBE) is characterised by progressive bilateral ophthalmoparesis and ataxia with impaired consciousness and/or pyramidal signs. ${ }^{12}$ A Japanese survey estimated the annual incidence of BBE as 0.078/100 000, with an annual onset of 100 cases. $^{2}$ The pathophysiology of the condition is not yet fully understood, but is associated with an autoimmune mechanism triggered by an antecedent infection. The majority of case reports of BBE have described the typical features of confusion or drowsiness. However, this is the first report of a patient with BBE presenting with pseudobulbar affect.

\section{CASE PRESENTATION}

A 36-year-old man with no medical history presented to his general practitioner feeling generally unwell with a 2-day history of increasing unsteadiness on walking. Eight days prior to admission, he had an episode of diarrhoea, which he attributed to food from an Indian takeaway. He denied any features of headache, neck stiffness or photophobia. He had not travelled out of the country recently. The family reported that his mood had become increasingly labile over the preceding 2 days.

On physical examination, the patient was feverish at $38^{\circ} \mathrm{C}$. Neurological examination revealed normal tone, power, reflexes and sensation in his upper and lower limbs. His plantar reflexes were upgoing. Examination of the cranial nerves revealed bilateral sluggish pupillary response to light and an ophthalmoplegia, with limitation of eye movements in all directions. There was ataxia of upper and lower limbs and his speech was slurred. Pseudobulbar affect was noted: the patient was tearful one moment and laughing the next. $\mathrm{He}$ experienced episodes of drowsiness throughout the day and his Glasgow Coma Scale score oscillated between 15/15 and 13/15 (eye opening to speech, disoriented). The patient appeared confused during conversation and was frequently disorientated to time, place and/or person. There was no evidence of dysarthria, tremor, nausea or vomiting.

\section{INVESTIGATIONS}

Routine blood tests were all within normal ranges including C reactive protein (CRP) of $2.3 \mathrm{mg} / \mathrm{L}$, white cell count (WCC) $6.3 \times 10^{9} / \mathrm{L}$ and neutrophil count $3.8 \times 10^{9} / \mathrm{L}$. Antinuclear antibody screen was negative. A lumbar puncture was performed which demonstrated a raised red blood cells of 4000/ $\mathrm{mm}^{3}$, with a normal protein, WCC, glucose and lactate. An Indian ink stain was negative. A cerebral CT angiogram and CT of his chest, abdomen and pelvis, which was undertaken with a differential diagnosis of paraneoplastic encephalomyelitis in mind, were reported as normal. MRI of the head was also performed, which demonstrated no abnormalities on T2 and fluid attenuated inversion recovery imaging in the brain or upper spinal cord.

Viral PCR for cytomegalovirus, Epstein-Barr virus and enterovirus, human herpesvirus 6, varicella-zoster virus and herpes DNA was negative. Serology for Brucella, Cryptococcus and Borrelia burgdorferi and antiganglioside antibodies was also negative. The patient eventually tested positive for Campylobacter IgG serology with an equivocal result for Campylobacter IgA.

\section{DIFFERENTIAL DIAGNOSIS}

Viral encephalitis and Miller-Fisher syndrome (MFS) were initially considered. However, acellular cerebrospinal fluid (CSF) and a negative viral screen make a diagnosis of encephalitis unlikely. The normal reflexes are inconsistent with MFS.

A wide differential was further considered including an autoimmune process, Lyme disease, cerebral lymphoma and paraneoplastic encephalomyelitis. As mentioned above, a negative autoimmune screen, negative Borrelia antibodies, unremarkable CSF and a normal MRI ruled out these differential diagnoses. Antiganglioside antibodies (anti-GQ1b) are often associated with BBE. Our patient was anti-GQ1b negative; however, the serum GQ1b IgG antibody-positive rate for BBE is only $70 \%$.

A diagnosis of $\mathrm{BBE}$ was made based on the clinical features of ataxia, ophthalmoplegia and impaired consciousness after Campylobacter infection.

\section{TREATMENT}

The patient was treated with intravenous immunoglobulins and plasmapheresis.

\section{OUTCOME AND FOLLOW-UP}

The patient made a complete recovery and was discharged 3 weeks after admission with no neurological sequelae.

\section{DISCUSSION}

BBE was described in 1950s by Edwin Bickerstaff ${ }^{4}$ as 'a grave syndrome with benign prognosis'. $\mathrm{He}$ 
reported a syndrome of ophthalmoplegia, ataxia and drowsiness, preceded by infection. ${ }^{5} 6$ Similarities were made with MFS and Guillain-Barré syndrome (GBS), including areflexia and a raised protein in the CSF. This prompted speculation as to a shared aetiology and in light of the common association with antecedent infection, an immune-based mechanism was proposed. Bickerstaff differentiated BBE from MFS by the presence of disturbed consciousness, which is only a feature in BBE. As a result, debate ensued as to the nature of the nerve damage in $\mathrm{BBE}$, which was regarded by Bickerstaff as a brainstem viral infection and by others as an autoimmune polyneuritis. ${ }^{7}$

Recently, Odaka et $a l^{8}$ proposed a set of clinical diagnostic criteria for the purpose of distinguishing BBE, MFS and GBS (table 1).

In general, patients are classified as having BBE rather than MFS if there is evidence of central involvement in the form of decreased consciousness or long tract signs, such as clonus, spasticity and hyper-reflexia. Odaka et $a l^{3}$ undertook a study to clarify the neurological features of 62 cases of BBE. Central Nervous System (CNS) manifestations included disturbed consciousness (drowsiness 45\%; stupor, semicoma or coma 29\%), hyperreflexia (34\%), Babinski's sign (40\%) and deep sensory impairment (16\%). Other common neurological features included facial weakness, bulbar palsy and nystagmus. There have also been case reports of patients with BBE presenting with hypersomnolence and decorticate posture, indicating CNS involvement. ${ }^{9}{ }^{10}$ However, to the best of our knowledge there have been no reports of patients with BBE demonstrating pseudobulbar affect-a prominent feature of our patient's symptomatology.

Although the pathophysiology of BBE is not yet fully understood, research has shed light on possible underlying mechanisms. Saito et $a l^{11}$ undertook a study to explain the phenotypic differences between MFS and BBE, based on the effects of sera in relation to the blood-brain barrier. Sera from patients with BBE disrupted the blood-brain barrier and were associated with increased secretion of matrix metalloproteinase secreted by human brain microvascular endothelial cells (BMECs), whereas sera from patients with MFS had no effect on BMECs. These findings may explain the CNS manifestations in patients with BBE.

Serum from patients with GBS, MFS and BBE has been shown to contain antibodies against gangliosides. ${ }^{8}$ BBE and MFS are associated with anti-GQ1b antibodies, whereas GBS is associated predominantly with anti-GM1 antibodies. Subsequently, it has been suggested that the three disorders form part of a continuous clinical spectrum, involving the peripheral nervous system and CNS. Notably, not all cases of BBE are anti-GQ1b positive; the serum GQ1b IgG antibody-positive rate in BBE is reported as

\section{Table 1 Proposed clinical diagnostic criteria for BBE, MFS and GBS}

\begin{tabular}{ll}
\hline Syndrome & Diagnostic criteria \\
\hline BBE & $\begin{array}{l}\text { Ophthalmoplegia and ataxia with disturbed consciousness and/or } \\
\text { pyramidal signs }\end{array}$ \\
MFS & $\begin{array}{l}\text { Acute ophthalmoplegia and ataxia with areflexia or hyporeflexia } \\
\text { GBS }\end{array}$ \\
\hline
\end{tabular}

BBE, Bickerstaff's brainstem encephalitis; MFS, Miller-Fisher syndrome; GBS, Guillain-Barré syndrome.
$70 \% .^{3}$ In comparison, GQ1b antibodies are positive in $83-100 \%$ of patients with MFS $^{12-14}$ and $8 \%$ with GBS. ${ }^{15}$

The presence of autoantibodies against anti-GQ1b is associated with ophthalmoplegia. The third, fourth and sixth cranial nerves contain a high concentration of GQ1b ganglioside, and neuromuscular blockade has been induced by anti-GQ1b IgG from patients with MFS. ${ }^{16}{ }^{17}$ In addition to ophthalmoplegia, BBE, MFS and GBS often involve a generalised absence of reflexes. This has been attributed to the loss of proprioceptive input from muscle spindles in patients with MFS. ${ }^{18}$ Liu et al demonstrated that anti-GQ1b antibody bound the vast majority of neuromuscular junctions (NMJ) of human extraocular muscles as well as nerve terminals inside muscle spindles. In contrast, anti-GQ1b ganglioside antibody binding to NMJs in human limb and axial muscle was sparse. ${ }^{19}$ Thus, the distribution of anti-GQ1b binding sites provides one explanation for the overlapping features of areflexia and ophthalmoparesis.

Our patient was anti-GQ1b negative with preserved reflexes, which is predictable in light of the above evidence. However, he also presented with ophthalmoplegia, suggesting other factors, aside from anti-GQ1b antibodies, are involved in the localisation of the clinical features of these syndromes.

There is also a need to clarify whether the overlapping features of BBE, MFS and GBS are due to identical mechanisms. Odaka $e t \mathrm{al}^{3}$ classified patients with BBE presenting with symmetrical flaccid limb weakness as BBE with overlapping GBS. He attributed these signs to an acute motor axonal neuropathy, as evidenced by the results of electrophysiological studies. However, a recent report on BBE suggested that this may instead represent CNS pathology, namely an immune-medicated inflammatory disease affecting the white matter of the brain and spinal cord. ${ }^{20}$

In several cases of BBE, antecedent pathogens have been identified, substantiating evidence that antiganglioside antibodies work via molecular mimicry with infectious agents. ${ }^{21}$ They include herpes simplex virus, cytomegalovirus, Epstein-Barr virus, varicella-zoster virus, measles virus, Salmonella typhi, Mycoplasma pneumonia and Campylobacter jejuni enteritis. ${ }^{8}$ Our patient suffered from an episode of diarrhoea and subsequently tested positive for C. jejuni.

A definitive treatment for BBE has yet to be found. The established treatment is the same as that used in GBS: intravenous immunoglobulin and plasmapheresis, although more clinical trials are required to determine its effectiveness.

The majority of cases are self-limiting, as was the case with our patient. However, contrary to Bickerstaff's comments, not all follow a benign course. In 2013, Koga published a paper reviewing the clinical aspects of $\mathrm{BBE}$ based on a nationwide survey in Japan. He concluded that BBE consisted of typical and atypical cases; typical BBE was associated with a good recovery, whereas atypical BBE was characterised by delayed recovery and abnormal CSF and brain MRI findings. ${ }^{2}$

More research is needed to unravel the mechanisms of BBE, define its relation to MFS and GBS and determine the effectiveness of treatment, although this is limited by the rarity of the condition. The described patient's unusual presentation with labile mood or pseudobulbar affect, rather than decreased consciousness could indicate a further patient group along the spectrum of disease. 


\section{Learning points}

- Bickerstaff's brainstem encephalitis (BBE) is a rare condition that must be suspected in patients presenting with ataxia, ophthalmoplegia and central nervous system involvement, which may take the form of pseudobulbar affect, decreased consciousness and/or pyramidal signs.

- Miller-Fisher syndrome, Guillain-Barré syndrome and BBE can be regarded as part of the same spectrum of disease with an underlying autoimmune mechanism, often triggered by an antecedent infection, which requires further elucidation.

- Treatment comprises intravenous immunoglobulins and electrophoresis, with most patients making a good recovery.

\section{Competing interests None.}

Patient consent Obtained.

Provenance and peer review Not commissioned; externally peer reviewed.

\section{REFERENCES}

1 Koga M. A nationwide survey of patients with Bickerstaff brainstem encephalitis: diversity of underlying mechanism. Rinsho Shinkeigaku 2013;53:1322-4.

2 Koga M. Bickerstaff brainstem encephalitis: epidemiology, diagnosis, and therapy. Nihon Rinsho 2013;71:898-903.

3 Odaka M, Yuki N, Yamada M, et al. Bickerstaff's brainstem encephalitis: clinical features of 62 cases and a subgroup associated with Guillain-Barre syndrome. Brain 2003;126(Pt 10):2279-90.

4 Bickerstaff E. Brain-stem encephalitis: further observations on a grave syndrome with benign prognosis. BMJ 1957;ii:1384-7.

5 Bickerstaff E, Cloake PCP. Mesencephalitis and rhombencephalitis. BMJ $1951 ; 2: 77-81$

6 Bickerstaff ER. Brain-stem encephalitis; further observations on a grave syndrome with benign prognosis. BMJ 1957;1:1384-7.
7 Ropper AH. The CNS in Guillain-Barré syndrome. Arch Neurol 1983:40:397-8.

8 Odaka M, Yuki N, Hirata K. Anti-GQ1b IgG antibody syndrome: clinical and immunological range. J Neurol Neurosurg Psychiatry 2001;70:50-5.

9 Wakerley BR, Soon D, Chan YC, et al. Atypical Bickerstaff brainstem encephalitis: ataxic hypersomnolence without ophthalmoplegia. J Neurol Neurosurg Psychiatry 2013:84:1206-7.

10 Shimozono K, Shimono K, Kusunoki S. Case of Bickerstaff brainstem encephalitis associated with spindle coma and decorticate posture. Rinsho Shinkeigaku 2012;52:656-9.

11 Saito K, Shimizu F, Koga M, et al. Blood-brain barrier destruction determines Fisher/ Bickerstaff clinical phenotypes: an in vitro study. J Neurol Neurosurg Psychiatry 2013:84:756-65.

12 Ito M, Kuwabara S, Odaka M, et al. Bickerstaff's brainstem encephalitis and Fisher syndrome from a continuous spectrum: clinical analysis of 581 cases. J Neurol 2008;255:674-82

13 Chiba A, Kusunoki S, Obata $H$, et al. Serum anti GQ1b lgG antibody is associated with ophthalmoplegia in Miller Fisher syndrome and Guillain-Barre syndrome: clinical and immunohistochemical studies. Neurology 1993:43:1911-17.

14 Willison HJ, Veitch J. Immunoglobulin subclass distribution and binding characteristics of anti-GQ1b antibodies in Miller Fisher syndrome. J Neuroimmunol 1994;50:159-65.

15 Kim JK, Bae JS, Kim DS, et al. Prevalence of anti-ganglioside antibodies and their clinical correlates with Guillain-barré syndrome in Korea: a nationwide multicenter study. J Clin Neurol 2014;10:94-100.

16 Buchwald B, Dudel J, Toyka KV. Neuromuscular blockade by immunoglobulin G from patients with Miller Fisher syndrome. Ann N Y Acad SCi 1998;841:659-69.

17 Chiba A, Kusunoki S, Obata $H$, et al. Serum anti-GQ1b lgG antibody is associated with ophthalmoplegia in Miller Fisher syndrome and Guillain-Barre syndrome: clinical and immunohistochemical studies. Neurology 1993;43:1911-17.

18 Kuwabara S, Asahina M, Nakajima M, et al. Special sensory ataxia in Miller Fisher syndrome detected by postural body sway analysis. Ann Neurol 1999;45:533-6

19 Liu JX, Willison HJ, Pedrosa-Domellöf F. Immunolocalization of GQ1b and related gangliosides in human extraocular neuromuscular junctions and muscle spindles. Invest Ophthalmol Vis Sci 2009;50:3226-32.

20 Roos R, Soliven B, Goldenberg F, et al. An elderly patient with Bickerstaff brainstem encephalitis and transient episodes of brainstem dysfunction. Arch Neurol 2008:65:821-4.

21 Yuki N. Anti-ganglioside antibody and neuropathy: review of our research. J Peripher Nerv Syst 1998;3:3-18.

Copyright 2014 BMJ Publishing Group. All rights reserved. For permission to reuse any of this content visit

http://group.bmj.com/group/rights-licensing/permissions.

BMJ Case Report Fellows may re-use this article for personal use and teaching without any further permission.

Become a Fellow of BMJ Case Reports today and you can:

- Submit as many cases as you like

- Enjoy fast sympathetic peer review and rapid publication of accepted articles

- Access all the published articles

- Re-use any of the published material for personal use and teaching without further permission

For information on Institutional Fellowships contact consortiasales@bmjgroup.com

Visit casereports.bmj.com for more articles like this and to become a Fellow 